\title{
miRNA-205-5p functions as a tumor suppressor by negatively regulating VEGFA and PI3K/Akt/mTOR signaling in renal carcinoma cells
}

\author{
JIANJUN HUANG ${ }^{1,2}$, XUE WANG ${ }^{1,2}$, GUOBING WEN $^{1,2}$ and YU REN ${ }^{1,2}$ \\ ${ }^{1}$ Department of Urologic Surgery, Ningbo Urology and Nephrology Hospital; ${ }^{2}$ Laboratory of Kidney Carcinoma, \\ Urology and Nephrology Institute of Ningbo University, Ningbo, Zhejiang 315000, P.R. China
}

Received September 14, 2018; Accepted March 13, 2019

DOI: $10.3892 /$ or.2019.7307

\begin{abstract}
MicroRNAs (miRNAs) are involved in the development of various types of cancers. Dysregulation of miR-205-5p has been reported in various types of human cancer. However, little is known concerning the role of miR-205-5p in renal cell carcinoma (RCC). The pr esent study was designed to investigate the role of miR-205-5p in RCC. The expression of miR-205-5p was measured in clear cell renal cell carcinoma (ccRCC) tissues and cell lines using RT-qPCR. RCC cell lines were transfected with miR-205-5p mimics. CCK-8 assays, wound healing assays, Matrigel invasion assays and nucleosome ELISAs were used to assess the effects of miR-205-5p on cell growth, migration, invasion and apoptosis, respectively. Western blotting was employed to detect changes in protein levels. Bioinformatic analyses and luciferase reporter assays were performed to identify the potential targets of miR-205-5p. Mouse xenograft models were used to verify the effect of miR-205-5p in vivo. The expression of miR-205-5p was found to be downregulated in $25 \mathrm{RCC}$ tissues compared to that noted in the adjacent normal tissues. Decreased expression of miR-205-5p was associated with poor clinical outcomes. Based on the results of the in vitro experiments, overexpression of miR-205-5p reduced RCC cell proliferation, invasion and migration. Overexpression of miR-205-5p also promoted apoptosis and inhibited the EMT in RCC cells. Moreover, the PI3K/Akt signaling pathway was found to be negatively regulated by miR-205-5p. Bioinformatic analyses and luciferase reporter assays revealed that $\mathrm{miR}-205-5 \mathrm{p}$ directly targeted the 3'-UTR of vascular endothelial growth factor A (VEGFA). Furthermore, miR-205-5p negatively regulated the expression of VEGFA in ccRCC cell lines. In ccRCC tissues, miR-205-5p
\end{abstract}

Correspondence to: Dr Yu Ren, Department of Urologic Surgery, Ningbo Urology and Nephrology Hospital, 1 Qianhe Road, Ningbo, Zhejiang 315000, P.R. China

E-mail: nbrenyu@163.com

Key words: miR-205-5p, RCC, PI3K/Akt, VEGFA, renal carcinoma cells expression was inversely correlated with VEGFA expression. Moreover, overexpression of miR-205-5p inhibited RCC growth in vivo in a mouse xenograft model. Overall, miR-205-5p functions as a tumor suppressor in RCC by targeting VEGFA and the PI3K/Akt signaling pathway, providing a potential therapeutic target for the treatment of ccRCC.

\section{Introduction}

Renal cell carcinoma ( $\mathrm{RCC}$ ) is a common cancer that accounts for $2-3 \%$ of all cancers worldwide, and clear cell renal cell carcinoma (ccRCC) is the most common histological subtype. Notably, ccRCC accounts for $\sim 75 \%$ of all RCCs and is characterized by a loss of chromosome $3 p$ in over $90 \%$ of all cases (1). After surgical resection, local recurrence or metastases occurs in $20-50 \%$ of patients diagnosed with localized RCC tumors, and the 5-year survival rate is $<10 \%$ (2). Currently, the molecular mechanisms underlying the progression of RCC are largely unknown. Thus, studies designed to elucidate the mechanisms of RCC and identify novel biomarkers are important to improve the clinical outcomes of RCC.

MicroRNAs (miRNAs) are a class of endogenous small non-coding RNAs that post-transcriptionally repress the expression of target genes by inhibiting the translation or promoting the degradation of mRNAs (3). Dysregulation of miRNAs has been shown to play vital roles in the tumorigenesis and development of various types of cancer, including ccRCC (4). Screening of the miRNA expression profile is one of the advanced strategies used to study tumor-associated molecules. Notably, miRNAs are highly conserved among different species and regulate various biological functions in an epigenetic manner (5). Importantly, miRNAs function as oncogenes or tumor-suppressor genes in various types of cancer and play significant roles in tumor development, suppression, metastasis and sensitivity or resistance to chemotherapy (6). Therefore, investigation of the biological functions of dysregulated miRNAs in RCC may contribute to the identification of novel biomarkers and therapeutic strategies for patients with RCC.

Recently, miR-205-5p has been reported to be involved in the tumorigenesis of various types of cancer, such as colon, prostate and hepatocellular carcinomas (7-10). For example, 
miR-205-5p has been reported to inhibit zinc finger E-box binding homeobox 1 (ZEB1), a transcription factor, in prostate cancer (10). Additionally, miR-205-5p was found to downregulate PTEN expression and thereby contribute to cisplatin resistance in ovarian cancer cells (11). However, the expression and function of miR-205-5p in RCC remain elusive. In the present study, miR-205-5p was found to be downregulated in RCC tissues and cell lines compared to corresponding normal tissues and cells. According to the results of the Kaplan-Meier analysis, low levels of miR-205-5p predicted a poor prognosis for patients with RCC. Overexpression of miR-205-5p inhibited the proliferation, migration, invasion and EMT of RCC cells. Bioinformatic analyses revealed that vascular endothelial growth factor A (VEGFA) is a direct downstream target of miR-205-5p. The expression of VEGFA in RCC tissues was negatively correlated with the miR-205-5p level. Furthermore, the PI3K/Akt/mTOR signaling pathway was inhibited in cells overexpressing miR-205-5p. In vivo experiments also confirmed that miR-205-5p inhibited the growth of xenograft tumors in mice. Based on our findings, miR-205-5p suppresses the tumorigenicity of RCC cells by targeting VEGFA and suppressing the PI3K/Akt/mTOR signaling pathway.

\section{Materials and methods}

Tissue collection. Twenty-five pairs of human RCC and adjacent normal tissues were surgically collected from patients at Ningbo Urology and Nephrology Hospital from March, 2015 to December 2017. Among the 25 enrolled patients, 12 were male, 13 were female and the mean age was $62.4 \pm 5.5$ years. Before surgery, none of the patients received any chemotherapy or radiotherapy. The clinicopathological features were recorded based on the American Joint Committee on Cancer (AJCC) standards (12). All patients provided written informed consent and the study was approved by the Ethics Committee of Ningbo Urology and Nephrology Hospital (Ningbo, China).

Cell culture and cell viability assay. Cells (293) were purchased from the Shanghai Institute for Biological Sciences (Shanghai, China). Human RCC cells (786-O, ACHN and Caki-1) were obtained from the American Type Culture Collection (ATCC; Manassas, VA, USA). 786-O and Caki-1 cells were maintained in RPMI-1640 medium (Gibco; Thermo Fisher Scientific, Inc., Waltham, MA, USA). ACHN and 293 cells were maintained in Dulbecco's modified Eagle's medium (DMEM; Gibco; Thermo Fisher Scientific, Inc.). Media were supplemented with $10 \%$ fetal bovine serum (FBS; Gibco Thermo Fisher Scientific, Inc.), $1 \%$ antibiotics (100 $\mu \mathrm{l} / \mathrm{ml}$ penicillin and $100 \mathrm{mg} / \mathrm{ml}$ streptomycin sulfate; Gibco; Thermo Fisher Scientific, Inc.) and $1 \%$ glutamine (Gibco; Thermo Fisher Scientific, Inc.). Cells were cultured in a humidified incubator containing $5 \% \mathrm{CO}_{2}$ at a temperature of $37^{\circ} \mathrm{C}$. The Cell Counting Kit-8 (CCK-8) assay was performed to assess cell viability. Briefly, cells (5,000 cells/well) were seeded in a 96-well plate. Twenty-four hours after transfection, $10 \mu \mathrm{l}$ of CCK-8 solution (Beyotime Institute of Biotechnology, Shanghai, China) was added, and $1 \mathrm{~h}$ later, the optical density (OD) value of each well was measured with an ELISA microplate reader (Bio-Rad Laboratories Inc., Hercules, CA, USA) at a wavelength of $595 \mathrm{~nm}$. Wells without cells were used as blanks. The experiments were performed in triplicate and repeated at least three times.

RNA purification and RT-PCR. Total RNA was extracted from the tissue samples and cells using TRIzol (Invitrogen; Thermo Fisher Scientific, Inc.), and purified with the RNeasy Maxi kit (Qiagen, Inc., Santa Clarita, CA, USA) according to the manufacturer's guidelines. RNA concentrations were measured using a NanoDrop 2000/2000c spectrophotometer (Thermo Fisher Scientific, Inc.). Reverse transcription to prepare cDNA templates was performed using a TaqMan MicroRNA Reverse Transcription kit (Applied Biosystems; Thermo Fisher Scientific, Inc.). Then, qPCR was performed with a miScript SYBR-Green PCR kit (Qiagen) and the LightCycler 480 Real-Time PCR system (Roche Diagnostics, Basel, Switzerland). GAPDH and U6 were used as internal controls for VEGFA and miR-205-5p, respectively. The following thermocycling conditions were used: $95^{\circ} \mathrm{C}$ for $1 \mathrm{~min}$, then 40 cycles of $95^{\circ} \mathrm{C}$ for $15 \mathrm{sec}, 55^{\circ} \mathrm{C}$ for $30 \mathrm{sec}$ and $70^{\circ} \mathrm{C}$ for $30 \mathrm{sec}$. The expression levels in tissues and cells were calculated using the $2^{-\Delta \Delta \mathrm{Cq}}$ method (13).

Cell transfection. Synthesized miR-205-5p mimics (5'-UCC UUCAUUCCACCGGAGUCUG-3') or the negative control (miR-NC) (5'-UCACAACCUCCUAGAAAGAGUAGA-3') were purchased from Suzhou GenePharma Co., Ltd. (Suzhou, China). The myr-Akt vector and empty vector were generous gifts from Dr Rui Yu (Ningbo University, Ningbo, China). Cells $\left(2 \times 10^{5}\right)$ were transfected with $20 \mu \mathrm{M}$ miRNA mimics or $2 \mu \mathrm{g}$ vector plasmid. Twenty-four hours after transfection, the cells were collected and assayed. Transfection was performed using Lipofectamine 2000 (Invitrogen; Thermo Fisher Scientific, Inc.), according to the manufacturer's instructions.

Wound healing and cell invasion assays. Cells were seeded in a 6-well plate and allowed to grow to $90 \%$ confluence to assess migration in vitro. Twenty-four hours after transfection, an artificial wound was created with a $200-\mu 1$ pipette tip in the center of the confluent cell monolayer. Then, the cells were cultured for another $24 \mathrm{~h}$ and the closure of the wound in each group was evaluated at the magnification of x2,000 under an inverted microscope (Olympus Corp., Tokyo, Japan). For the invasion assay, $1 \times 10^{5}$ cells were suspended in $200 \mu \mathrm{l}$ of serum-free medium and plated in upper Transwell chambers (Costar; Corning Inc., Corning, NY, USA) coated with Matrigel (BD Biosciences, Franklin Lakes, NJ, USA). Six hundred microliters of serum-containing medium were added to the bottom well. After culture in a humidified atmosphere containing $5 \% \mathrm{CO}_{2}$ at $37^{\circ} \mathrm{C}$ for $24 \mathrm{~h}$, the cells that had migrated and adhered to the lower surface of the membrane were fixed with $70 \%$ methanol and stained with $1 \%$ crystal violet for $15 \mathrm{~min}$. Finally, the number of cells that migrated across the membrane were counted. Migration and invasion of cells was evaluated with an inverted Olympus phase-contrast microscope (x200 magnification) (Olympus Corp.). Each experiment was performed in triplicate.

Apoptosis assay. The percentage of apoptotic cells was measured using Nucleosome ELISA kit (cat. no. 11544675001; Roche Diagnostics), according to the manufacturer's 
instructions. Briefly, cells were seeded into 24-well plates and transfected. Twenty-four hours later, the cells were collected and lysed. A biotin-labeled mouse antibody against histone 3 that specifically binds to the histone component of captured nucleosomes derived from apoptotic cells was used in this assay. The bound antibody was detected following an incubation with horseradish peroxidase (HRP)-conjugated streptavidin at room temperature for $1 \mathrm{~h}$. HRP catalyzes the conversion of the colorless tetramethylbenzidine (TMB) to produce a blue color. The addition of stop solution turns the solution yellow, and the intensity was proportional to the number of nucleosomes in the sample. Each experiment was performed in triplicate.

Western blot assay. Cells were lysed in RIPA buffer (Beyotime Institute of Biotechnology). The protein concentration was determined by the Bradford assay kit according to the manufacturer's guidelines (Beyotime Institute of Biotechnology). Lysates were centrifuged at $12,000 \times \mathrm{g} 5 \mathrm{~min}$ at $4^{\circ} \mathrm{C}$ and stored at $-70^{\circ} \mathrm{C}$. Equal amounts $(20 \mu \mathrm{g})$ of protein lysates were separated on $10 \%$ SDS-PAGE gels and electrophoretically transferred onto polyvinylidene difluoride (PVDF) membranes (Millipore, Minneapolis, MN, USA). PVDF membranes were blocked with $10 \%$ skim milk powder dissolved in TBST buffer at room temperature for $1 \mathrm{~h}$. Then, PVDF membranes were stripped and washed with TBST, and then incubated with a primary antibody (dilution of 1:1,000) in TBST containing $5 \%$ bovine serum albumin (BSA) overnight at $4^{\circ} \mathrm{C}$. Primary antibodies against the following proteins were used: MMP-7 (cat. no. ab207299), MMP-9 (cat. no. ab73734), Snail (cat. no. ab53519) (all from Abcam, Cambridge, MA, USA), $\mathrm{N}$-cadherin (cat. no. 4061), caspase-3 (cat. no. 9662), Bcl-2 (cat. no. 3498), Bcl-xL (cat. no. 2762), p-PI3K (cat. no. 4228), t-PI3K (cat. no. 4225), p-Akt (cat. no. 4058), t-Akt (cat. no. 4691), p-mTOR (cat. no. 5536), t-mTOR (cat. no. 2983) (Cell Signaling Technology, Danvers, USA) and GAPDH (Sigma-Aldrich; Merck KGaA, Darmstadt, Germany). Next, membranes were washed with TBST three times and incubated with an HRP-conjugated anti-rabbit (cat. no. RABHRP1) or anti-mouse secondary antibody (cat. no. RABHRP2) (Sigma-Aldrich; Merck KGaA) for $1 \mathrm{~h}$ at room temperature. Signals were visualized using an ECL reagent (Pierce; Thermo Fisher Scientific, Inc.). All western blots were replicated in three times and the intensity of the western blot signals was analyzed using ImageJ software version 1.48 (NIH; National Institutes of Health, Bethesda, MD, USA).

Luciferase reporter assay. The potential miR-205-5p binding sites in the VEGFA 3'-UTR were predicted using TargetScan 7.1 software (www.targetscan.org/). Sequences containing the wild-type (VEGFA-wt) or mutant (VEGFA-mut) seed region of VEGFA were synthesized and cloned into a luciferase reporter plasmid (pMIR-REPORT) (Promega Corporation, Madison, WI, USA). The VEGFA-wt or VEGFA-mut plasmids and miR-205-5p or miR-NC were co-transfected using Lipofectamine 2000 (Invitrogen; Thermo Fisher Scientific, Inc.), according to the manufacturer's instructions. Twenty-four hours after transfection, firefly and Renilla luciferase activities were measured using the Dual-Luciferase assay (Promega Corporation) according to the manufacturer's instructions. All luciferase assays were performed in triplicate.
In vivo experiments. The animal study was approved by the Ethics Committee on Animal Research of Ningbo Urology and Nephrology Hospital (Ningbo, China). Male nude mice (BALB/c, 4-5 weeks old) were purchased from the Shanghai Laboratory Animal Center, Shanghai Institute for Life Sciences, Chinese Academy of Sciences (Shanghai, China). The total number of mice were 50 and the weight of the mice was $20 \pm 5 \mathrm{~g}$. The mice were housed in a facility at $23-24^{\circ} \mathrm{C}$, and the light-dark cycle was set at 12 -h intervals. The miR-205-5p-overexpressing 786-O cells or miR-NC-overexpressing 786-O cells were prepared by transfecting the cells with a recombinant lentivirus carrying the miR-205-5p precursor sequence or a scrambled control, respectively (Shanghai GenePharma Co., Ltd., Shanghai, China). Cells were suspended in $100 \mu \mathrm{l}$ of phosphate-buffered saline (PBS) and subcutaneously injected into the flank of a nude mouse. The width and length of the tumor nodules were measured every three days. The volume of subcutaneous tumors was calculated as tumor volume $=$ length width $\mathrm{x}$ width $/ 2$. At 31 days post-injection, the mice were removed from their cages and gently restrained while resting on the benchtop. Cervical dislocation was performed manually and resulted in euthanasia within $\sim 10 \mathrm{sec}$ and the tumors were excised for use in subsequent experiments.

Statistical analysis. All statistical analyses were conducted using the SPSS 20.0 software (IBM Corp., Armonk, NY, USA). All measurement data are presented as means \pm standard deviations (SDs). Univariate Cox proportional hazards regression analyses were performed to identify any significant variables predicting survival status. Multivariate Cox proportional hazard analyses were performed to assess the independent predictors of survival. One-way analysis of variance (ANOVA) with post hoc Tukey's test was chosen to compare data between multiple groups and paired Student's t-test was used to compare data between two groups. $\mathrm{P}<0.05$ was considered indicative of a significant difference.

\section{Results}

Expression of miR-205-5p is downregulated in RCC and correlates with the outcomes of patients with RCC. We examined the endogenous expression of miR-205-5p in RCC tissues $(n=25)$ and adjacent normal kidney tissues $(n=25)$ to determine whether miR-205-5p is dysregulated in RCC. Significantly lower expression of miR-205-5p was detected in RCC tumor tissues than that observed in the normal tissues $(\mathrm{P}<0.01$; Fig. 1A). In addition, we measured the expression of miR-205-5p in the cell line 293 and RCC cell lines 786-O, Caki-1 and ACHN. Significantly lower expression of miR-205-5p was observed in RCC cell lines than in the 293 cells (Fig. 1B). Then, we divided the 25 samples into a high miR-205-5p expression group $(n=13)$ and low miR-205-5p expression group $(n=12)$ according the median expression to further evaluate the relationship between miR-205-5p expression and the clinicopathological features of RCC. As shown in Table I, a lower miR-205-5p level was associated with an advanced tumor stage, Fuhrman stage and more lymph node metastasis, but was not associated with age, sex or tumor size. In addition, the Kaplan-Meier analysis revealed that 

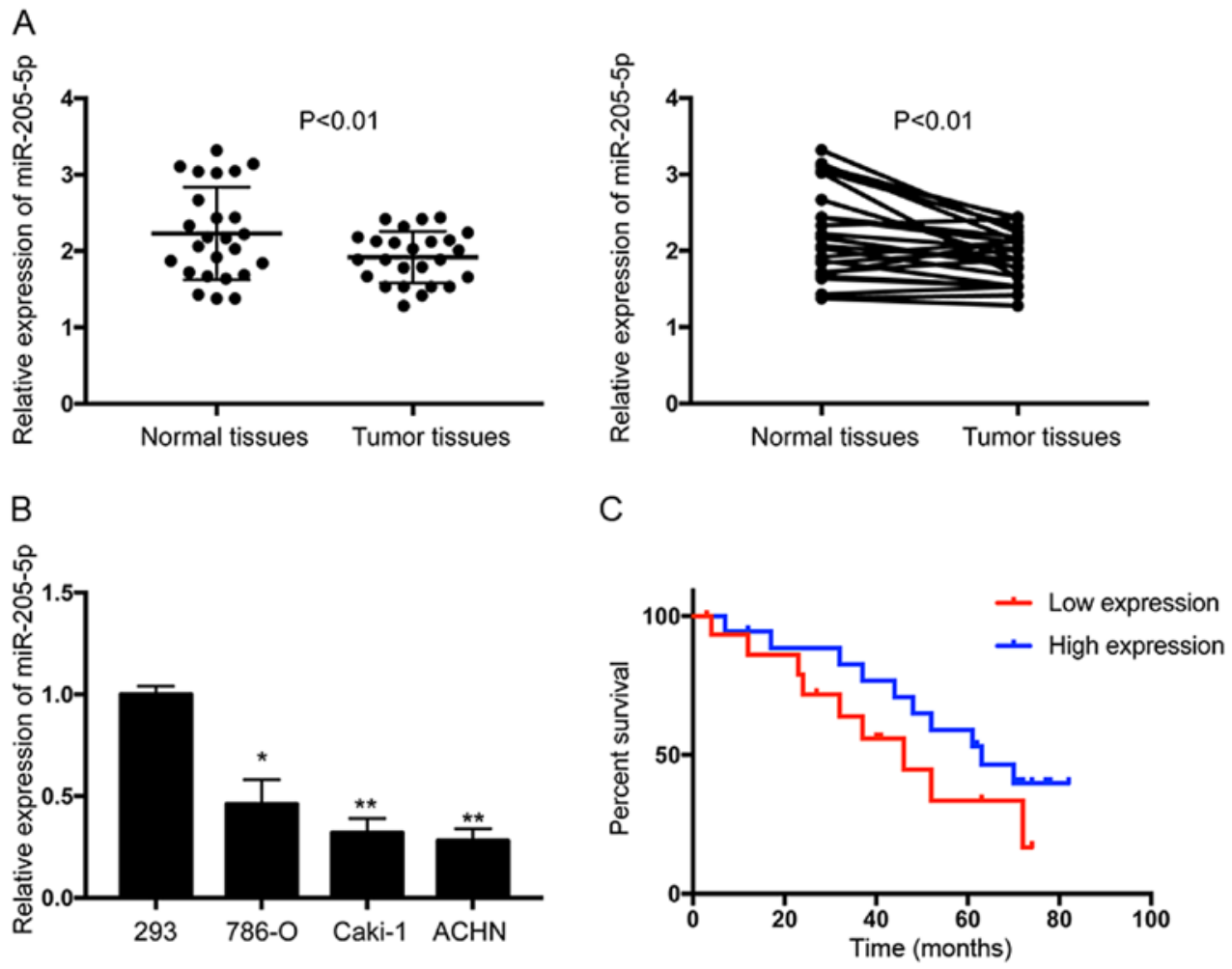

Figure 1. Expression of miR-205-5p is downregulated in RCC tissues and cell lines and is correlated with patient survival. (A) The expression of miR-205-5p in 25 RCC tissues compared with adjacent normal tissues were analyzed using RT-qPCR. (B) The expression of miR-205-5p in RCC cell lines (786-O, Caki-1 and ACHN) and 293 cells. (C) The correlation between miR-205-5p expression and the survival of patients with RCC. ${ }^{*} \mathrm{P}<0.05$ and ${ }^{* *} \mathrm{P}<0.01$; the data are presented as the means \pm standard deviations (SD) from triplicate measurements. RCC, renal cell carcinoma.

downregulation of miR-205-5p was associated with a poor prognosis, indicating that miR-205-5p may serve as a promising candidate prognostic biomarker for patients with RCC (mean follow-up time of 42 months) (Fig. 1C). A univariate analysis using the Cox proportional hazard regression model revealed statistically significant correlations between the overall survival of patients with miR-205-5p expression $(\mathrm{P}=0.017)$ and tumor stage $(\mathrm{P}=0.023)$ (Table II). A multivariate analysis including miR-205-5p expression and the tumor stage showed that miR-205-5p $(\mathrm{P}=0.024)$ and tumor stage $(\mathrm{P}=0.034)$ were independent prognostic factors for the overall survival of patients with RCC (Table II).

miR-205-5p inhibits the proliferation and migration of RCC cells. The 786-O and ACHN cells were transfected with the miR-205-5p mimic or miR-NC to investigate the function of miR-205-5p in RCC. We performed RT-qPCR to analyze the miR-205-5p expression in the transfected cells. The expression of miR-205-5p was significantly upregulated in both $786-\mathrm{O}(\mathrm{P}<0.001)$ and ACHN $(\mathrm{P}<0.001)$ cells at $24 \mathrm{~h}$ after transfection (Fig. 2A). The CCK-8 assay was employed to evaluate cell proliferation. Overexpression of miR-205-5p significantly suppressed the proliferation of both 786-O and ACHN cells compared with cells transfected with miR-NC (Fig. 2B). Thus, miR-205-5p suppressed the proliferation of RCC cells. Then, a scratch assay was performed to assess cell migration. Based on the results of the scratch assay, the migratory distance was markedly reduced in both cell lines transfected with the miR-205-5p mimic (Fig. 2C). Thus, miR-205-5p suppresses the migration of RCC cells.
Table I. Correlation between miR-205-5p expression and the clinicopathological features of the RCC patients.

\begin{tabular}{lcccc}
\hline & \multicolumn{3}{c}{ miR-205-5p levels } & \\
Parameters & Cases & Low & High & P-value \\
\hline Sex & & & & 0.320 \\
$\quad$ Male & 13 & 8 & 5 & \\
Female & 12 & 5 & 7 & \\
Age (years) & & & & 0.513 \\
$\quad<60$ & 10 & 6 & 4 & \\
$\geq 60$ & 15 & 7 & 8 & \\
Tumor size (cm) & & & & 0.561 \\
$<4$ & 11 & 5 & 6 & \\
$\geq 4$ & 14 & 8 & 6 & \\
Tumor stage & & & & 0.009 \\
$\quad$ T1-T2 & 12 & 3 & 9 & \\
T3-T4 & 13 & 10 & 3 & \\
Fuhrman grade & & & & 0.025 \\
I-II & 9 & 2 & 7 & \\
$\quad$ III-IV & 16 & 11 & 5 & \\
Lymph node metastasis & & & & 0.008 \\
$\quad$ Yes & 11 & 9 & 2 & \\
No & 14 & 4 & 10 & \\
\hline
\end{tabular}

RCC, renal cell carcinoma. 
Table II. Univariate and multivariate analyses of the clinicopathological factors for overall survival in the RCC cases.

\begin{tabular}{|c|c|c|c|c|c|c|}
\hline \multirow[b]{2}{*}{ Variables } & \multicolumn{3}{|c|}{ Univariate analysis } & \multicolumn{3}{|c|}{ Multivariate analysis } \\
\hline & HR & $95 \% \mathrm{CI}$ & P-value & $\mathrm{HR}$ & $95 \% \mathrm{CI}$ & P-value \\
\hline miR-205-5p expression (high vs. low) & 0.382 & $0.147-0.683$ & 0.017 & 0.398 & $0.215-0.842$ & 0.024 \\
\hline Sex (male vs. female) & 1.732 & $0.422-2.531$ & 0.142 & & & \\
\hline Age (<60 vs. $\geq 60$ years) & 0.973 & $0.684-2.023$ & 0.783 & & & \\
\hline Tumor size (<4 vs. $\geq 4 \mathrm{~cm})$ & 2.054 & $0.563-3.127$ & 0.426 & & & \\
\hline Tumor stage (T1-T2 vs. T3-T4) & 2.128 & $1.378-5.241$ & 0.023 & 2.351 & $1.942-4.336$ & 0.034 \\
\hline Fuhrman grade (I-II vs. III-IV) & 2.52 & $0.768-3.082$ & 0.613 & & & \\
\hline Lymph node metastasis (yes vs. no) & 3.83 & $0.542-4.117$ & 0.532 & & & \\
\hline
\end{tabular}

RCC, renal cell carcinoma; HR, hazard ratio; CI, confidence interval.

Overexpression of miR-205-5p inhibits invasion and epithelial-mesenchymal transition (EMT) and induces apoptosis in RCC cells. Next, we assessed the effects of miR-205-5p on the invasion of RCC cells using Transwell assays. As shown in Fig. 3A, overexpression of miR-205-5p decreased the invasive ability of both 786-O and ACHN cell lines. Meanwhile, the levels of invasion and migration marker proteins (MMP-7 and MMP-9) were also obviously decreased in cells overexpressing miR-205-5p (Fig. 3B). Since epithelial-mesenchymal transition (EMT) is an indispensable process for tumor cell invasion and migration (14), we examined whether miR-205-5p affects EMT in RCC cells. As shown in Fig. 3B, overexpression of miR-205-5p significantly decreased the protein levels of $\mathrm{N}$-cadherin, $\beta$-catenin and Snail. Based on these data, miR-205-5p may inhibit the migration of RCC cells by suppressing the EMT. We also analyzed the numbers of apoptotic cells and found that overexpression of miR-205-5p significantly increased the percentage of apoptotic 786-O and ACHN cells (Fig. 3C). Furthermore, the levels of apoptosis-related proteins were examined using western blotting. As indicated in Fig. 3D, caspase-3, Bcl-2 and Bcl-xL levels were reduced in cells overexpressing miR-205-5p. Therefore, miR-205-5p likely suppresses the proliferation of RCC cells by inducing apoptosis.

miR-205-5p increases the chemosensitivity of RCC cells. RCC is normally resistant to conventional cytotoxic chemotherapies. Therefore, we investigated the effect of miR-205-5p on the responses of RCC cells to various chemotherapeutic agents. First, we tested paclitaxel, an agent known to inhibit cell division and thereby induce cell death. Measurements of the half maximal inhibitory concentration $\left(\mathrm{IC}_{50}\right)$ showed an increased sensitivity of 786-O and ACHN cells to paclitaxel (Fig. 4A). Similar results were also obtained with 5-FU and oxaliplatin, agents that block DNA replication (Fig. 4B and C). We also tested sunitinib, a receptor tyrosine kinase (RTK) inhibitor used to treat patients with advanced ccRCC (15). Notably, overexpression of miR-205-5p also increased the sensitivity of 786-O and ACHN to sunitinib (Fig. 4D). Therefore, miR-205-5p is also involved in the response of RCC cells to chemotherapy.
miR-205-5p inactivates the PI3K/Akt/mTOR signaling pathway in RCC cells. The PI3K/Akt/mTOR signaling pathway exerts significant effects on the proliferation, invasion and apoptosis of RCC cells (16). We, therefore, investigated the effects of miR-205-5p on the PI3K/Akt signaling pathway. As indicated in Fig. 5A, overexpression of miR-205-5p markedly decreased p-PI3K, p-Akt and p-mTOR levels. Constitutively active Akt (myr-Akt) was overexpressed as described in a previous study to further elucidate the role of the PI3K/Akt pathway in the antitumor effects of miR-205-5p (17). Overexpression of myr-Akt successfully mimicked the activation of Akt (Fig. 5B). Myr-Akt also significantly suppressed miR-205-5p-induced apoptosis (Fig. 5C). Based on these data, the tumor suppressor function of miR-205-5p in RCC is likely and at least partially mediated by its repression of the PI3K/Akt/mTOR pathway.

VEGFA is a direct target of miR-205-5p. According to bioinformatic analysis tools (TargetScan; www.targetscan.org and miRanda; www.microrna.org), we speculated that VEGFA is a candidate target of miR-205-5p. As shown in Fig. 6A, the RT-qPCR analysis revealed significantly decreased levels of the VEGFA mRNA in 786-O and ACHN cells transfected with miR-205-5p. Western blot analyses also showed decreased levels of the VEGFA protein in 786-O and ACHN cells transfected with the miR-205-5p mimic (Fig. 6B). We performed a Dual-Luciferase assay to further confirm that VEGFA is a target of miR-205-5p. The wild-type (WT) and mutant (Mut) VEGFA 3'-untranslated region (3'-UTR) constructs were subcloned into the pMIR reporter plasmid. Overexpression of miR-205-5p decreased luciferase activity in both 786-O and ACHN cells transfected with the wild-type (WT) 3'-UTR of VEGFA, but not the mutant (Mut) 3'-UTR (Fig. 6D). Levels of the VEGFA mRNA in RCC and normal adjacent tissues were examined using qRT-PCR to elucidate the clinical relevance of miR-205-5p-mediated targeting of VEGFA in RCC. As shown in Fig. 6E, the VEGF mRNA was expressed at much higher levels in RCC tumor tissues than that noted in normal tissues. Furthermore, an inverse correlation was observed between the expression of miR-205-5p and VEGFA expression in RCC tissues ( $r=-0.179$ ) (Fig. 6F). These results further confirmed that VEGFA expression is negatively regulated 

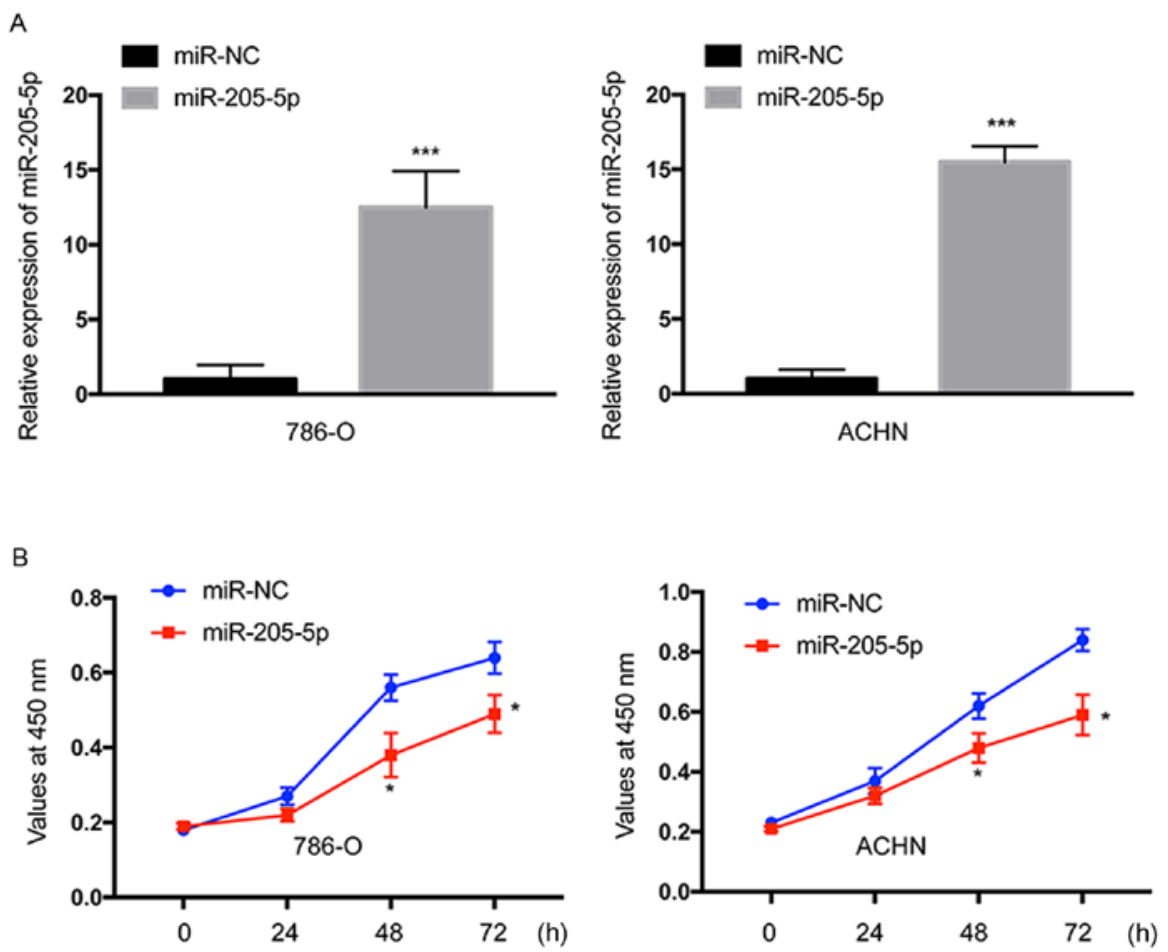

C

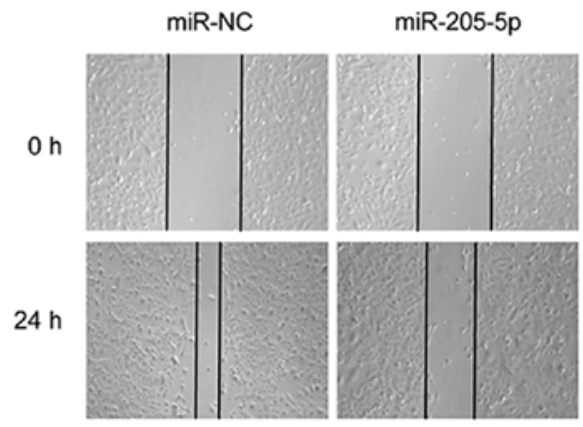

$786-0$

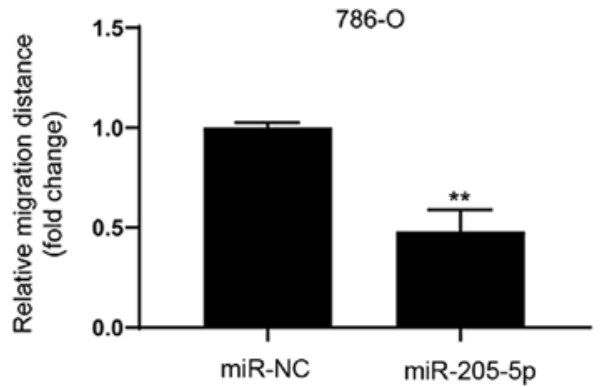

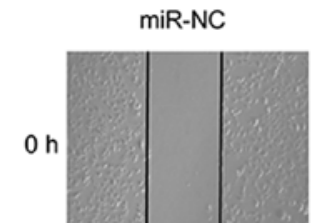

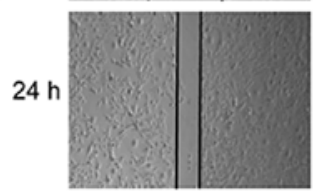

$\mathrm{ACHN}$
miR-205-5p
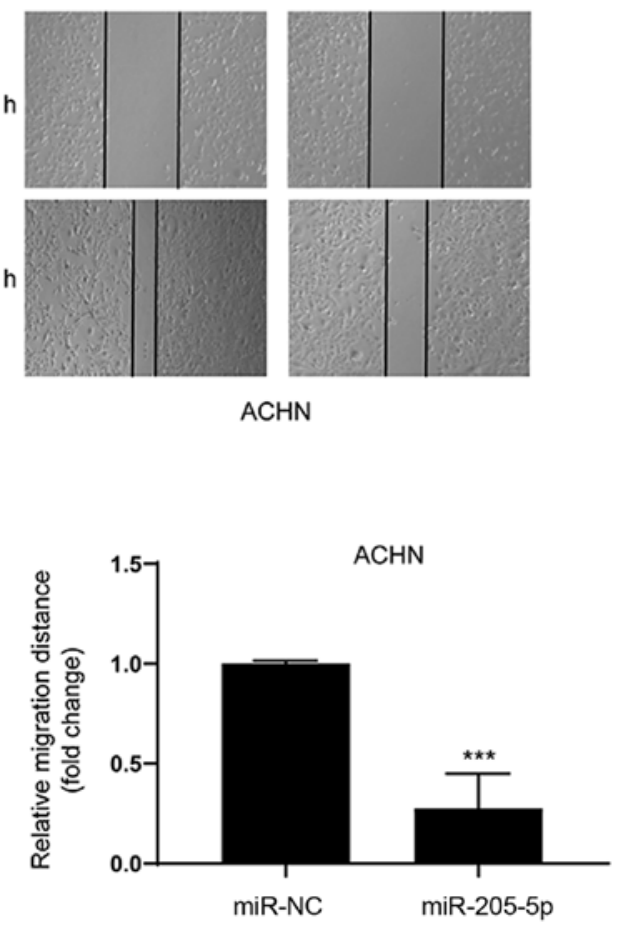

Figure 2. miR-205-5p inhibits the proliferation and migration of RCC cells. (A) Analysis of miR-205-5p expression in transfected 786-O and ACHN cells using qPCR. (B) Effect of miR-205-5p overexpression on the proliferation of 786-O and ACHN cells, as assessed using the CCK-8 assay. (C) Results of the scratch assay using 786-O and ACHN cells overexpressing miR-205-5p. ${ }^{*} \mathrm{P}<0.05,{ }^{* * *} \mathrm{P}<0.01$ and ${ }^{* * * *} \mathrm{P}<0.001$; data are presented as the means \pm standard deviations (SD) of triplicate measurements. RCC, renal cell carcinoma; CCK-8; Cell Counting Kit-8.

by miR-205-5p in RCC. In summary, VEGFA is a target of miR-205-5p in RCC.

miR-205-5p suppresses RCC cell tumorigenesis in vivo. We stably expressed miR-NC or miR-205-5p in 786-O cells and then subsequently implanted these cells into both posterior flanks of nude mice to investigate whether overexpression of miR-205-5p attenuates the progression of RCC in vivo. Tumor sizes were measured after 7 days. From the 22 nd to the 31 st day, the miR-NC group developed significantly larger tumors 


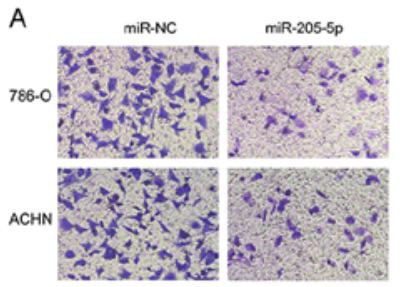

B
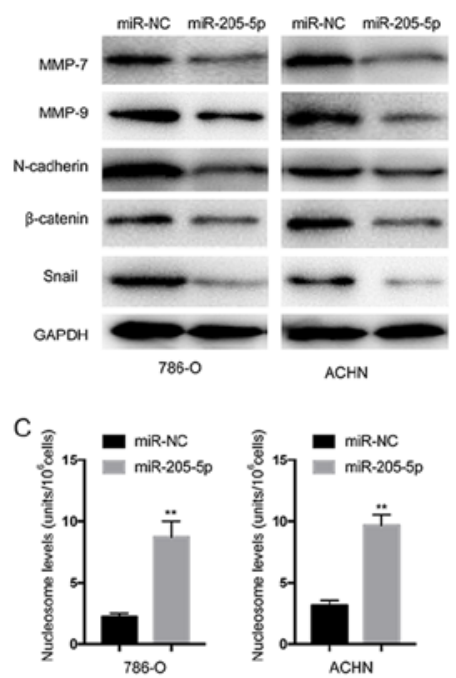
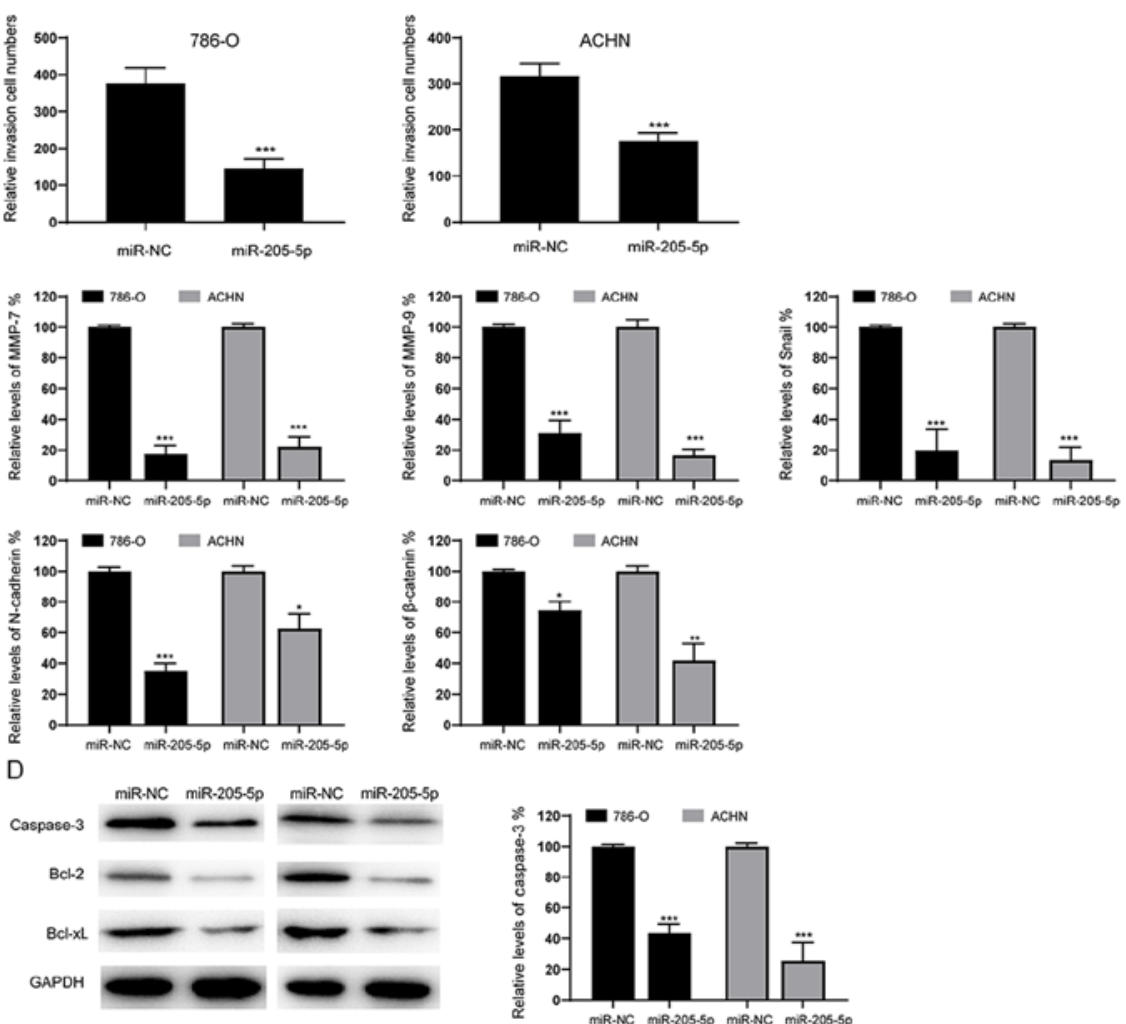

$786-0$

$\mathrm{ACHN}$

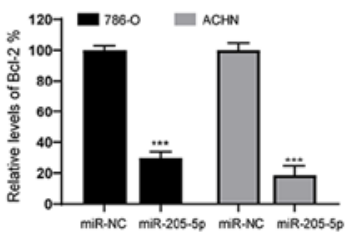

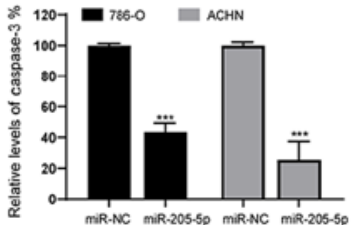

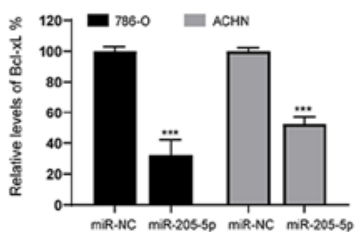

Figure 3. Overexpression of miR-205-5p inhibits invasion and EMT and induces apoptosis in RCC cells. (A) A Transwell assay was used to investigate the effect of miR-205-5p overexpression on the invasion of 786-O and ACHN cells. (B) After overexpression of miR-205-5p, total cell lysates were collected and subjected to western blotting with the indicated antibodies. (C) A nucleosome ELISA assay was performed to investigate the effect of miR-205-5p overexpression on the apoptosis of 786-O and ACHN cells. (D) After overexpression of miR-205-5p, total cell lysates were collected and subjected to western blotting with the indicated antibodies. ${ }^{*} \mathrm{P}<0.05,{ }^{* * *} \mathrm{P}<0.01$ and ${ }^{* * * *} \mathrm{P}<0.001$; data are presented as the means \pm standard deviations (SD) of triplicate measurements. RCC, renal cell carcinoma; EMT, epithelial-mesenchymal transition.
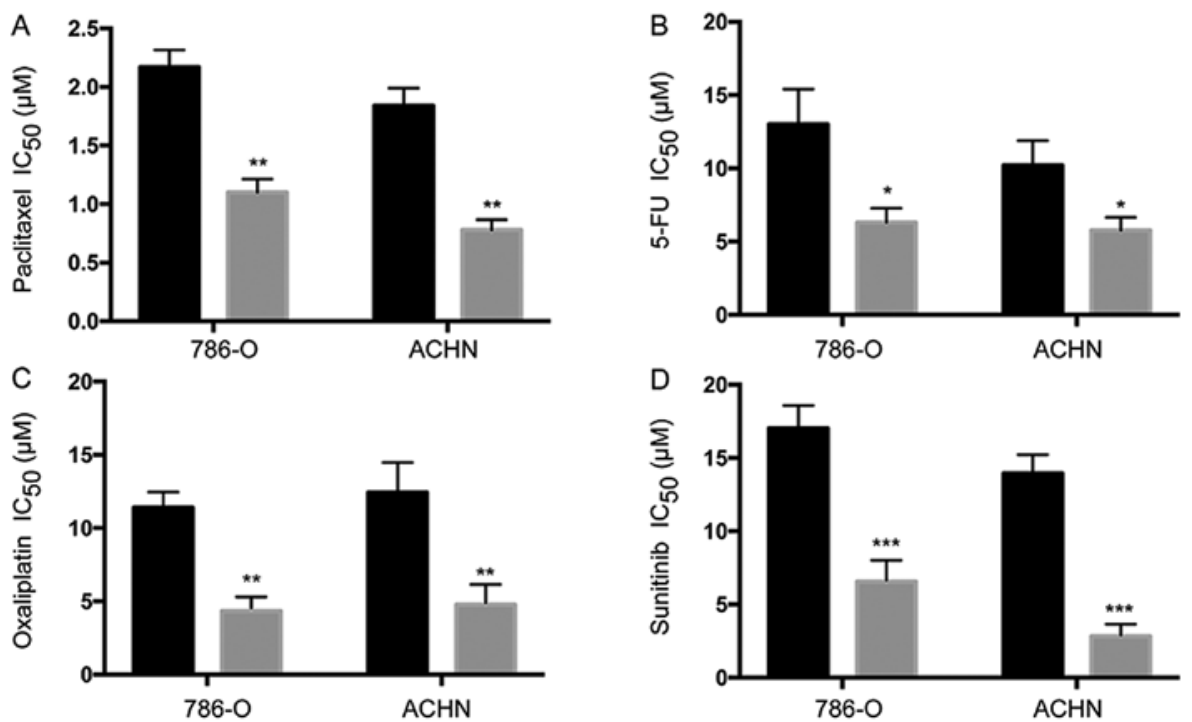

Figure 4. Overexpression of miR-205-5p sensitizes RCC cell lines to paclitaxel, 5-FU, oxaliplatin and sunitinib. After the transfection of miR-205-5p or miR-NC mimics, 786-O and ACHN cells were incubated with (A) paclitaxel, (B) 5-FU, (C) oxaliplatin, or (D) sunitinib. IC 50 $_{50}$ values were calculated after $48 \mathrm{~h}$ of drug treatment using the CCK- 8 assay. ${ }^{*} \mathrm{P}<0.05,{ }^{* * *} \mathrm{P}<0.01$ and ${ }^{* * * *} \mathrm{P}<0.001$; the data are presented as the means \pm standard deviations (SDs) of triplicate measurements. RCC, renal cell carcinoma 5-FU, 5-fluorouracil. 

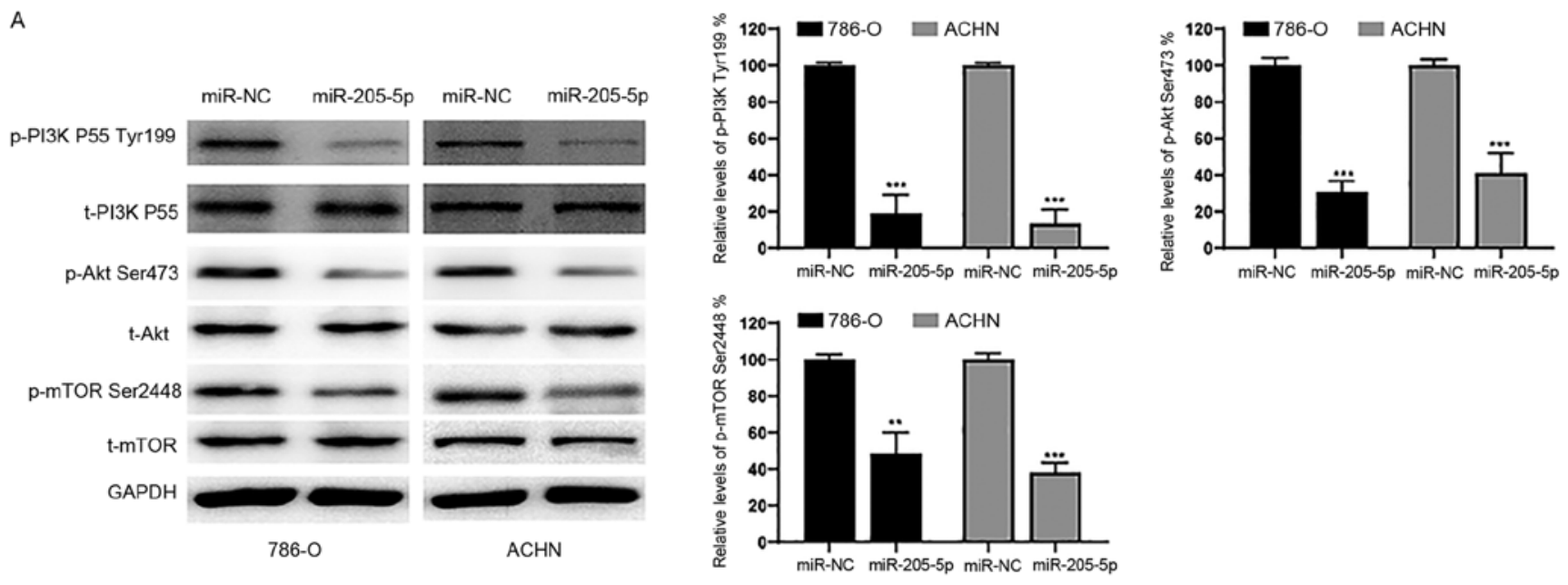

B
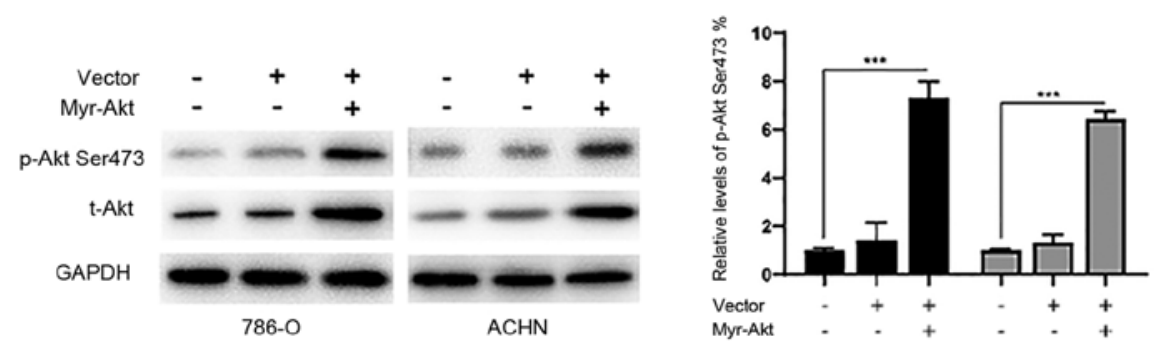

C

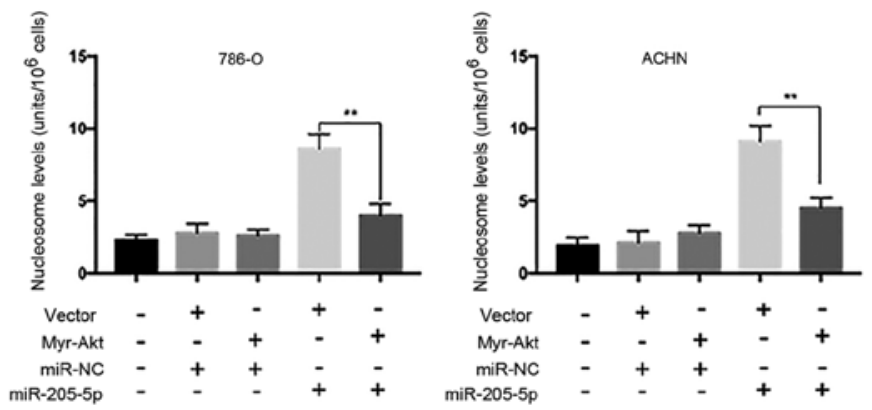

Figure 5. Effect of miR-205-5p on the PI3K/Akt/mTOR signaling pathway. (A) The 786-O and ACHN cells were transfected with miR-205-5p for 24 h, and then p-PI3K, t-PI3K, p-Akt, t-Akt, p-mTOR, t-mTOR and GAPDH levels were determined using western blotting. (B) The 786-O and ACHN cells were treated with the indicated reagents for $24 \mathrm{~h}$, and then p-Akt, t-Akt and GAPDH levels were determined using western blotting. (C) The 786-O and ACHN cells were treated with the indicated reagents for $24 \mathrm{~h}$, and then a nucleosome ELISA was performed to measure apoptosis. ${ }^{* * *} \mathrm{P}<0.01$ and ${ }^{* * * *} \mathrm{P}<0.001$; data are presented as the means \pm standard deviations (SD) of triplicate measurements.

than the miR-205-5p group (Fig. 7A). Meanwhile, the final tumor weight of the miR-NC group was much greater than the miR-205-5p group (Fig. 7B). Consistent with the results from the in vitro studies, lower levels of the caspase- $3, \mathrm{Bcl}-2$ and $\mathrm{Bcl}-\mathrm{xL}$ proteins were detected in the tumor tissues from the miR-205-5p-expressing group than in the miR-NC-expressing group (Fig. 7C). Based on these data, miR-205-5p also inhibits tumor growth in vivo.

\section{Discussion}

In the present study, we identified a novel molecular mechanism by which miR-205-5p modulates renal cell carcinoma (RCC) through the suppression of VEGFA expression and the PI3K/Akt/mTOR signaling pathway. Based on accumulating evidence, microRNAs (miRNAs) play vital roles in tumorigenesis, and the expression of various miRNAs correlates with clinical characteristics and outcomes. Numerous studies have investigated the biological roles of miRNAs in RCC. For example, miR-30a-5p was found to suppress the proliferation and promote the apoptosis of RCC cells by targeting GRP78 (18). Moreover, miR-720 targets the E-cadherin- $\alpha /$ E-catenin complex and plays a clinically significant role in RCC (19). In addition, miR-30b-5p inhibits the proliferation, metastasis and epithelial to mesenchymal transition (EMT) of RCC cells by repressing G-protein subunit $\alpha-13$ (20). Thus, miRNAs play important roles in RCC progression by regulating various biological activities of cells.

In the present study, we confirmed significantly lower miR-205-5p expression in RCC tissues and cell lines than these levels in their normal counterparts. Notably, miR-205-5p has been shown to be downregulated and function as a tumor 


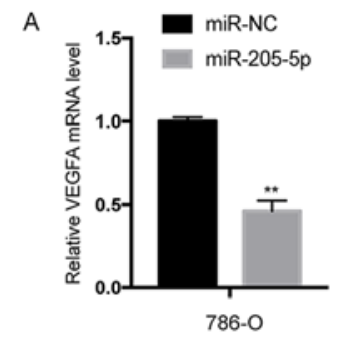

C

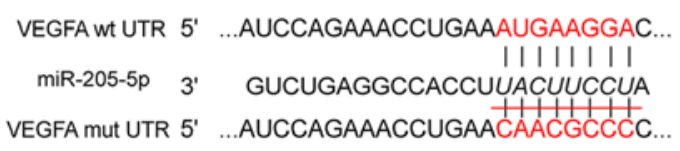

$\mathrm{E}$

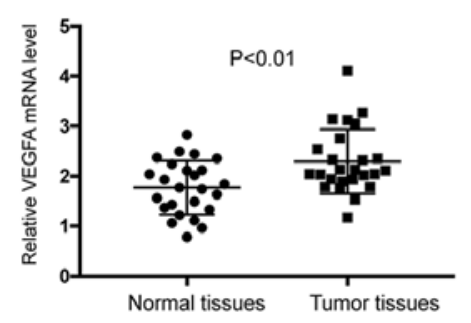

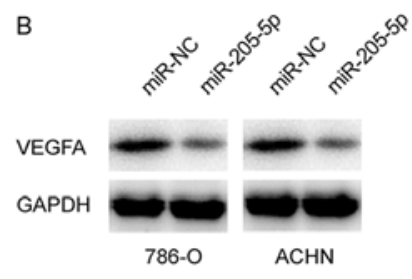

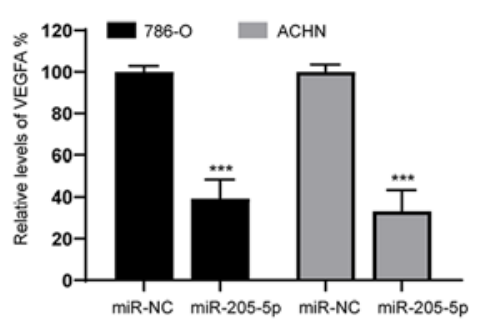

$\mathrm{D}$
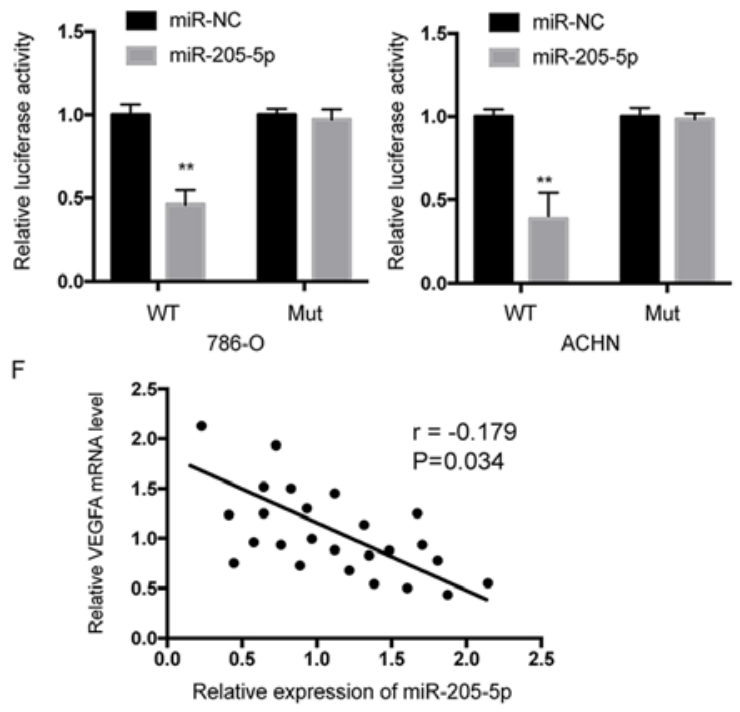

Figure 6. VEGFA is a direct target of miR-205-5p, and VEGFA expression is inversely correlated with miR-205-5p expression in RCC tissues. (A) RT-qPCR analysis of VEGFA mRNA expression in transfected 786-O and ACHN cells. (B) Western blot analysis of levels of the VEGFA protein in transfected 786-O and ACHN cells. (C) The predicted miR-205-5p binding site and corresponding mutation in the 3'-UTR of the VEGFA mRNA. (D) Luciferase activities of wild-type and the mutant pmir-VEGFA 3'-UTR reporter in 786-O and ACHN cells. (E) RT-qPCR analysis of VEGFA mRNA levels in RCC and adjacent normal tissues. (F) Spearman's correlation analysis was performed to determine the correlation between miR-205-5p and VEGFA expression in RCC tissues. ${ }^{* *} \mathrm{P}<0.01$ and ${ }^{* * *} \mathrm{P}<0.001$; data are presented as the means \pm standard deviations (SD) of triplicate measurements. VEGFA, vascular endothelial growth factor A.

A

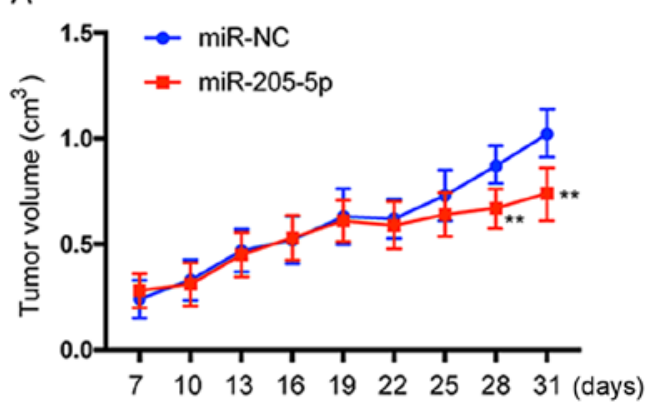

B

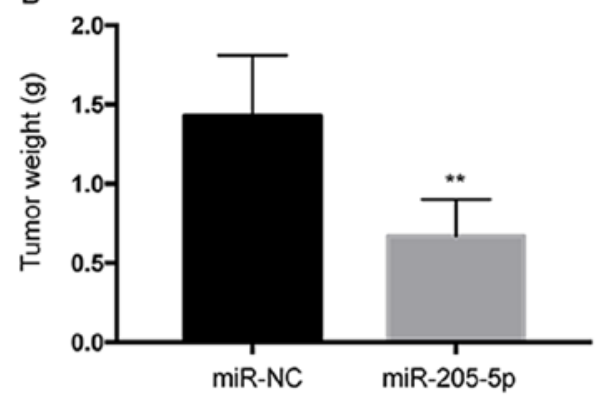

C
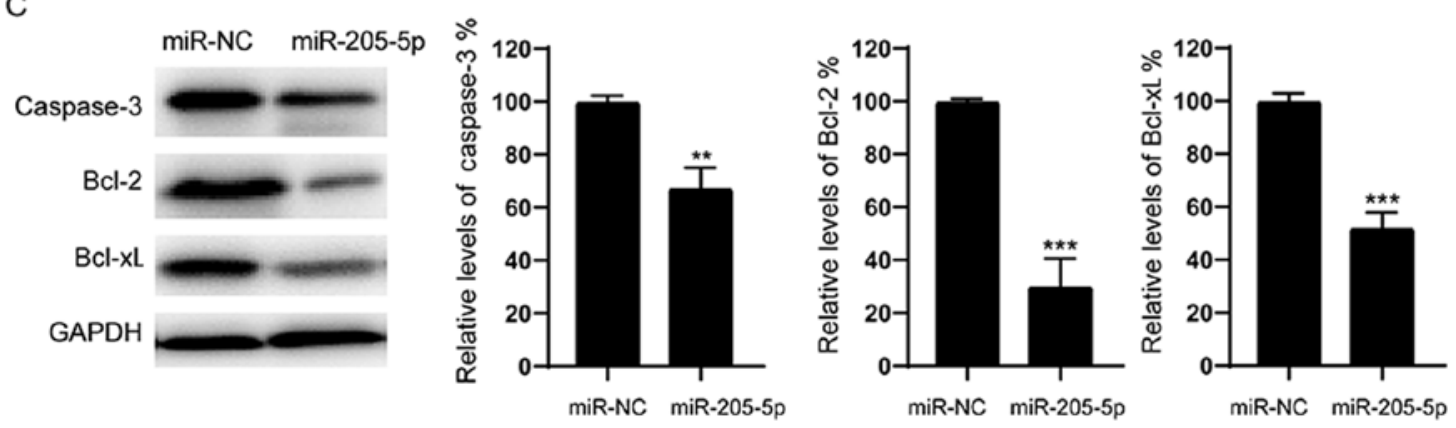

Figure 7. miR-205-5p inhibits tumor growth in vivo. BALB/c nude mice were subcutaneously injected with 786-O cells infected with lentiviruses harboring miR-NC or miR-205-5p. (A) Tumor volumes were monitored over time, as indicated in the figure. (B) Tumors were excised and weighed after 31 days. (C) Levels of the caspase-3, Bcl-2 and Bcl-xL proteins in the tumor tissues were determined using western blot assays. ${ }^{* *} \mathrm{P}<0.01$ and ${ }^{* * *} \mathrm{P}<0.001$; data are presented as the means \pm standard deviations (SDs) of triplicate measurements. 
suppressor in various types of cancer. For example, miR-205-5p expression was found to be significantly decreased in prostate cancer tissues and to inhibit cancer cell aggressiveness by targeting HMGB3 (9). In a recent study, miR-205-5p significantly suppressed the migration and invasion of oral squamous carcinoma cells by inhibiting TIMP-2 expression (21). However, miR-205-5p expression is significantly increased in non-small cell lung cancer tissues, and it functions as an oncogene by downregulating erbB3 expression (22). Notably, overexpression of miR-205-5p increased the chemosensitivity of RCC cells to various agents, such as paclitaxel, 5-FU, oxaliplatin and sunitinib, in the present study. Meanwhile, overexpression of miR-205-5p was found to mediate the resistance of hepatocellular carcinoma cells to 5-FU (8). These discrepancies may be due to the differences in cancer types. Since miRNAs regulate the expression of different target genes depending on specific cellular and disease context (3), investigation of the functions of miR-205-5p in additional cancer types is warranted.

Overexpression of miR-205-5p inhibited the proliferation, migration and invasion of RCC cells. Moreover, overexpression of miR-205-5p induced apoptosis, accompanied by the downregulation of caspase-3, Bcl-2 and Bcl-xL. Two different pathways lead to the apoptosis, namely, the extrinsic and intrinsic pathways (23). The intrinsic pathway is strictly regulated by Bcl-2 family members (24). Our data are consistent with a previous study showing that miR-205-5p modulated the levels of Bcl-2 proteins in melanoma (25). Thus, miR-205-5p may induce tumor cell apoptosis via the intrinsic pathway.

Multiple signaling pathways participate in modulating tumor progression, including the EMT signaling pathway (26). EMT plays a vital role in the metastasis of tumor cells. During the EMT process, the expression of E-cadherin, tight junction proteins and other epithelial markers are decreased in tumor cells, causing them to lose their epithelial characteristics (27). Meanwhile, the expression of mesenchymal markers, such as vimentin and $\mathrm{N}$-cadherin, are upregulated during EMT, leading to an increase in migratory and invasive behaviors (28). Based on accumulating evidence, miRNAs are key modulators of EMT in many types of cancer. Recently, overexpression of miR-205-5p was shown to inhibit EMT in colon cancer (7). According to Elgamal et al, overexpression of miR-205 decreases the protein levels of the mesenchymal markers N-cadherin, vimentin and ZEB1 in breast cancer cells (29). These findings are consistent with our findings. In the present study, miR-205-5p decreased the levels of mesenchymal marker proteins, such as N-cadherin, Snail and $\beta$-catenin. Therefore, miR-205-5p may suppress the invasion and migration of RCC cells by inhibiting EMT.

The PI3K/Akt/mTOR signaling pathway plays important roles in various significant biological processes, such as proliferation, development and apoptosis (30). Activity of the PI3K/Akt signaling pathway is relatively high in ccRCC among all cancer types, as indicated by increased levels of phosphorylated Akt and Akt substrates (31). Therefore, treatments targeting the PI3K/Akt/mTOR pathway represent a promising strategy to inhibit ccRCC (32). Recently, miRNAs have emerged as a new class of important regulators of the PI3K/Akt pathway. For example, miR-182-5p has been identified as a negative regulator of $\mathrm{AKT}$, and downregulation of miR-182-5p results in AKT activation and subsequent RCC proliferation (33). In contrast, miR-122 is a positive regulator of the $\mathrm{PI} 3 \mathrm{~K} / \mathrm{Akt}$ signaling pathway, promoting the proliferation, invasion and migration of RCC cells (34). In the present study, we investigated the effects of miR-205-5p on the PI3K/Akt/mTOR pathway and observed decreased phosphorylation of proteins involved in the PI3K/Akt/mTOR pathway. Notably, forced expression of constitutively activated Akt decreased miR-205-5p-induced apoptosis, suggesting that miR-205-5p at least partially exerts its antitumor effects by inhibiting the PI3K/Akt/mTOR signaling pathway. The inhibition of the PI3K/Akt/mTOR pathway was likely mediated by several possible mechanisms. For example, phosphatase and tensin homologue (PTEN) dephosphorylates phosphatidylinositol $(3,4,5)$-triphosphate (PIP3) and therefore inhibits the activation of the PI3K/Akt/mTOR signaling pathway. In the present study, VEGFA expression was downregulated by miR-205-5p. VEGFA activates the PI3K/Akt pathway $(35,36)$. Thus, we hypothesized that one mechanism by which miR-205-5p inactivated the PI3K/Akt pathway was through inhibition of VEGFA expression.

We applied bioinformatics tools to identify the potential target genes of miR-205-5p and clarify the mechanisms by which miR-205-5p inhibited tumor growth. Among the putative targets, we focused on VEGFA, since accumulating evidence indicates that VEGFA is associated with the proliferation and metastasis of RCC (37). VEGFA plays an important role in the response to angiogenesis during tumorigenesis (38). Consistent with a previous study, the study also validated VEGFA as a target of miR-205-5p using a luciferase reporter assay (39). Overexpression of miR-205-5p inhibited the expression of both the VEGFA mRNA and protein in 786-O and ACHN cells. Moreover, VEGFA expression was found to be inversely correlated with miR-205-5p expression in RCC tissues. Based on these data, VEGFA is a direct target gene of miR-205-5p in RCC. To note, we did not investigate the effects of VEGFA on RCC cells in our study, as previous studies have already investigated the functions of VEGFA which could exert antitumor effects against the $\operatorname{RCC}(40,41)$. According to previous studies, VEGFA has been described as an important determinant of the increase in the tumorigenicity of cells that undergo EMT in solid cancers $(42,43)$. Moreover, VEGFA has been demonstrated to increase the tumor-initiating stem cell population, to induced EMT (44). Furthermore, inhibition of VEGFA could lead to a decrease in EMT markers in cancer stem cells (45). Based on these findings, we hypothesized that VEGFA plays an essential role in the EMT process. Thus, the change in expression of EMT-related proteins in this study may be due to the inhibition of VEGFA by miR-205a and we will test this in future research.

In summary, miR-205-5p expression was downregulated in RCC tissues and cells. Ectopic expression of miR-205-5p inhibited the proliferation, migration and invasion of RCC cells and promoted apoptosis. Overexpression of miR-205-5p repressed the PI3K/Akt/mTOR pathway. Moreover, miR-205-5p inhibited RCC growth in vivo in a mouse xenograft model. In addition, we identified VEGFA as a target of miR-205-5p in RCC. To note, other possible targets of miR-205-5p may exist, and VEGFA could also be subject to the regulation of other miRNAs, since we found that overexpression of miR-205-5p 
could only partially inhibit the expression of VEGFA. To the best of our knowledge, this study is the first to explore the role of miR-205-5p in RCC. However, the regulatory mechanism of miR-205-5p remains elusive. Further studies of miR-205-5p in $\mathrm{RCC}$ are required to elucidate the complicated mechanisms of tumorigenesis.

\section{Acknowledgements}

Not applicable.

\section{Funding}

No funding was received.

\section{Availability of data and materials}

The datasets used in the present study are available from the corresponding author upon reasonable request.

\section{Authors' contributions}

JH performed most of the experiments and drafted the manuscript; XW performed some of the experiments; GW analyzed the data; YR designed the study and reviewed the manuscript. All authors read and approved the manuscript and agree to be accountable for all aspects of the research in ensuring that accuracy or integrity of any parts of the work are appropriately investigated and resolved.

\section{Ethics approval and consent to participate}

The clinicopathological features were recorded based on the American Joint Committee on Cancer (AJCC) standards. All patients provided written informed consent and the study was approved by the Ethics Committee of Ningbo Urology and Nephrology Hospital (Ningbo, China). The animal study was approved by the Ethics Committee on Animal Research of Ningbo Urology and Nephrology Hospital (Ningbo, China).

\section{Patient consent for publication}

Not applicable.

\section{Competing interests}

The authors state that they have no competing interests.

\section{References}

1. van den Berg E and Dijkhuizen T: Classification of renal cell cancer based on (cyto)genetic analysis. Contrib Nephrol 128: 51-61, 1999.

2. Capitanio U and Montorsi F: Renal cancer. Lancet 387: 894-906, 2016.

3. Bartel DP: MicroRNAs: Genomics, biogenesis, mechanism, and function. Cell 116: 281-297, 2004.

4. Zimmerman AL and Wu S: MicroRNAs, cancer and cancer stem cells. Cancer Lett 300: 10-19, 2011.

5. Tutar Y: miRNA and cancer; Computational and experimental approaches. Curr Pharm Biotechnol 15: 429, 2014.

6. Calin GA and Croce CM: MicroRNA signatures in human cancers. Nat Rev Cancer 6: 857-866, 2006.
7. Gulei D, Magdo L, Jurj A, Raduly L, Cojocneanu-Petric R, Moldovan A, Moldovan C, Florea A, Pasca S, Pop LA, et al: The silent healer: miR-205-5p up-regulation inhibits epithelial to mesenchymal transition in colon cancer cells by indirectly up-regulating E-cadherin expression. Cell Death Dis 9: 66, 2018.

8. Shao P, Qu WK, Wang CY, Tian Y, Ye ML, Sun DG, Sui JD, Wang LM, Fan R and Gao ZM: MicroRNA-205-5p regulates the chemotherapeutic resistance of hepatocellular carcinoma cells by targeting PTEN/JNK/ANXA3 pathway. Am J Transl Res 9: 4300-4307, 2017

9. Yamada Y, Nishikawa R, Kato M, Okato A, Arai T, Kojima S, Yamazaki K, Naya Y, Ichikawa T and Seki N: Regulation of $H M G B 3$ by antitumor miR-205-5p inhibits cancer cell aggressiveness and is involved in prostate cancer pathogenesis. J Hum Genet 63: 195-205, 2018.

10. Li L and Li S: miR-205-5p inhibits cell migration and invasion in prostatic carcinoma by targeting ZEB1. Oncol Lett 16: 1715-1721, 2018.

11. Shi X, Xiao L, Mao X, He J, Ding Y, Huang J, Peng C and Xu Z: miR-205-5p mediated downregulation of PTEN contributes to cisplatin resistance in $\mathrm{C} 13 \mathrm{~K}$ human ovarian cancer cells. Front Genet 9: 555, 2018.

12. Edge SB and Compton CC: The American joint committee on cancer: The 7th edition of the AJCC cancer staging manual and the future of TNM. Ann Surg Oncol 17: 1471-1474, 2010.

13. Livak KJ and Schmittgen TD: Analysis of relative gene expression data using real-time quantitative PCR and the $2^{-\Delta \Delta C \mathrm{~T}}$ method. Methods 25: 402-408, 2001.

14. Gonzalez DM and Medici D: Signaling mechanisms of the epithelial-mesenchymal transition. Sci Signal 7: re8, 2014.

15. Rodriguez-Vida A, Hutson TE, Bellmunt J and Strijbos MH: New treatment options for metastatic renal cell carcinoma. ESMO Open 2: e000185, 2017.

16. Duran I, Lambea J, Maroto P, González-Larriba JL, Flores L, Granados-Principal S, Graupera M, Sáez B, Vivancos A and Casanovas O: Resistance to targeted therapies in renal cancer: The importance of changing the mechanism of action. Target Oncol 12: 19-35, 2017.

17. Yu R, Yu BX, Chen JF, Lv XY, Yan ZJ, Cheng Y and Ma Q: Anti-tumor effects of Atractylenolide I on bladder cancer cells. J Exp Clin Cancer Res 35: 40, 2016.

18. Wang C, Cai L, Liu J, Wang G, Li H, Wang X, Xu W, Ren M, Feng L, Liu P, et al: MicroRNA-30a-5p inhibits the growth of renal cell carcinoma by modulating GRP78 expression. Cell Physiol Biochem 43: 2405-2419, 2017.

19. Bhat NS, Colden M, Dar AA, Saini S, Arora P, Shahryari V, Yamamura S, Tanaka Y, Kato T, Majid S, et al: MicroRNA-720 regulates E-cadherin- $\alpha$ E-catenin complex and promotes renal cell carcinoma. Mol Cancer Ther 16: 2840-2848, 2017.

20. Liu W, Li H, Wang Y,Zhao X, Guo Y, Jin J and Chi R: MiR-30b-5p functions as a tumor suppressor in cell proliferation, metastasis and epithelial-to-mesenchymal transition by targeting G-protein subunit $\alpha-13$ in renal cell carcinoma. Gene 626: 275-281, 2017.

21. Nagai H, Hasegawa S, Uchida F, Terabe T, Ishibashi Kanno N, Kato K, Yamagata K, Sakai S, Kawashiri S, et al: MicroRNA-205-5p suppresses the invasiveness of oral squamous cell carcinoma by inhibiting TIMP2 expression. Int J Oncol 52: 841-850, 2018.

22. Jiang M, Zhong T, Zhang W, Xiao Z, Hu G, Zhou H and Kuang H: Reduced expression of miR-205-5p promotes apoptosis and inhibits proliferation and invasion in lung cancer A549 cells by upregulation of ZEB2 and downregulation of erbB3. Mol Med Rep 15: 3231-3238, 2017.

23. Fulda S: Therapeutic opportunities based on caspase modulation. Semin Cell Dev Biol 82: 150-157, 2018.

24. Kale J, Osterlund EJ and Andrews DW: BCL-2 family proteins: Changing partners in the dance towards death. Cell Death Differ 25: 65-80, 2018.

25. Dahmke IN, Backes C, Rudzitis-Auth J, Laschke MW, Leidinger P, Menger MD, Meese E and Mahlknecht U: Curcumin intake affects miRNA signature in murine melanoma with mmu-miR-205-5p most significantly altered. PLoS One 8: $\mathrm{e} 81122,2013$.

26. Brabletz T: EMT and MET in metastasis: Where are the cancer stem cells? Cancer Cell 22: 699-701, 2012.

27. Brabletz T, Kalluri R, Nieto MA and Weinberg RA: EMT in cancer. Nat Rev Cancer 18: 128-134, 2018.

28. Kraljevic Pavelic S, Sedic M, Bosnjak H, Spaventi S and Pavelic K: Metastasis: New perspectives on an old problem. Mol Cancer 10: 22, 2011. 
29. Elgamal OA, Park JK, Gusev Y, Azevedo-Pouly AC, Jiang J Roopra A and Schmittgen TD: Tumor suppressive function of mir-205 in breast cancer is linked to HMGB3 regulation. PLoS One 8: e76402, 2013.

30. LoRusso PM: Inhibition of the PI3K/AKT/mTOR pathway in solid tumors. J Clin Oncol 34: 3803-3815, 2016.

31. Akbani R, Ng PK, Werner HM, Shahmoradgoli M, Zhang F, Ju Z, Liu W, Yang JY, Yoshihara K, Li J, et al: A pan-cancer proteomic perspective on The Cancer Genome Atlas. Nat Commun 5: 3887, 2014.

32. Porta C, Paglino C and Mosca A: Targeting PI3K/Akt/mTOR signaling in cancer. Front Oncol 4: 64, 2014

33. Xu X, Wu J, Li S, Hu Z, Xu X, Zhu Y, Liang Z, Wang X, Lin Y, Mao Y, et al: Downregulation of microRNA-182-5p contributes to renal cell carcinoma proliferation via activating the AKT/FOXO3a signaling pathway. Mol Cancer 13: 109, 2014.

34. Lian JH, Wang WH, Wang JQ, Zhang $\mathrm{YH}$ and $\mathrm{Li} \mathrm{Y}$ MicroRNA-122 promotes proliferation, invasion and migration of renal cell carcinoma cells through the PI3K/Akt signaling pathway. Asian Pac J Cancer Prev 14: 5017-5021, 2013.

35. Ruan GX and Kazlauskas A: Axl is essential for VEGF-Adependent activation of PI3K/Akt. EMBO J 31: 1692-1703, 2012

36. Chen CH, Lai JM, Chou TY, Chen CY, Su LJ, Lee YC, Cheng TS, Hong YR, Chou CK, Whang-Peng J, et al: VEGFA upregulates FLJ10540 and modulates migration and invasion of lung cancer via PI3K/AKT pathway. PLoS One 4: e5052, 2009.

37. Vachhani $P$ and George S: VEGF inhibitors in renal cell carcinoma. Clin Adv Hematol Oncol 14: 1016-1028, 2016.

38. Comunanza V and Bussolino F: Therapy for cancer: Strategy of combining anti-angiogenic and target therapies. Front Cell Dev Biol 5: 101, 2017.

39. An G, Liang S, Sheng C, Liu Y and Yao W: Upregulation of microRNA-205 suppresses vascular endothelial growth factor expression-mediated PI3K/Akt signaling transduction in human keloid fibroblasts. Exp Biol Med 242: 275-285, 2017.
40. Zeng FC, Zeng MQ, Huang L, Li YL, Gao BM, Chen JJ, Xue RZ and Tang ZY: Downregulation of VEGFA inhibits proliferation, promotes apoptosis, and suppresses migration and invasion of renal clear cell carcinoma. Onco Targets Ther 9: 2131-2141, 2016.

41. Chen YS, Meng F, Li HL, Liu QH, Hou PF, Bai J and Zheng JN: Dicer suppresses MMP-2-mediated invasion and VEGFA-induced angiogenesis and serves as a promising prognostic biomarker in human clear cell renal cell carcinoma. Oncotarget 7: 84299-84313, 2016.

42. Fantozzi A, Gruber DC, Pisarsky L, Heck C, Kunita A, Yilmaz M, Meyer-Schaller N, Cornille K, Hopfer U, Bentires-Alj M and Christofori G: VEGF-mediated angiogenesis links EMT-induced cancer stemness to tumor initiation. Cancer Res 74: 1566-1575, 2014.

43. Gonzalez-Moreno O, Lecanda J, Green JE, Segura V, Catena R, Serrano D and Calvo A: VEGF elicits epithelial-mesenchymal transition (EMT) in prostate intraepithelial neoplasia (PIN)-like cells via an autocrine loop. Exp Cell Res 316: 554-567, 2010.

44. Kim LC, Cook RS and Chen J: mTORC1 and mTORC2 in cancer and the tumor microenvironment. Oncogene 36: 2191-2201, 2017.

45. BesharatZM, Sabato C, Po A, Gianno F, Abballe L, Napolitano M, Miele E, Giangaspero F, Vacca A, Catanzaro G, et al: Low expression of miR-466f-3p sustains epithelial to mesenchymal transition in Sonic hedgehog medulloblastoma stem cells through Vegfa-Nrp2 signaling pathway. Front Pharmacol 9: 1281, 2018.

This work is licensed under a Creative Commons Attribution-NonCommercial-NoDerivatives 4.0 International (CC BY-NC-ND 4.0) License. 